Letter to the Editor

\title{
Prevalence of use and appropriateness of antidepressants prescription in acutely hospitalized elderly patients
}

Depression is often under-recognized in older patients, even if antidepressants (AD) are commonly prescribed, with a prevalence of use that increase with ageing [1]. Nevertheless, even if a diagnosis of depression is established, inappropriate treatment can occur [2]. Beers criteria are the most widely screening tools used to detect inappropriate prescription of drugs in people aged 65 years or more [3]. Since 2010, attempts to adapt the Beers' criteria have been made in Europe [4,5]. Tricyclic drugs are the ADs to be always avoided in the elderly, owing to their anticholinergic side effects, such as cognitive impairment, delirium, urinary retention and falls [3]. Selective serotonin reuptake inhibitors (SSRIs) should be used with caution because they can cause or exacerbate hyponatraemia [3]. To our knowledge no studies have been conducted on recent data collected in hospitalized older patients in order to assess the appropriateness of prescription of ADs. With this background, the objectives of this study were to investigate the prevalence of $\mathrm{AD}$ use and prescription appropriateness, according to the above-cited criteria, in a large cohort of older patients hospitalized in Italian internal medicine and geriatric wards from 2010 to 2017.

Data were obtained from the register REgistro POliterapie -Società Italiana Medicina Interna (REPOSI), an ongoing collaboration between the Italian Society of Internal Medicine (SIMI), IRCCS Fondazione Ca` Granda Ospedale Maggiore Policlinico and the Istituto di Ricerche Farmacologiche Mario Negri IRCCS. The REPOSI is a multicenter register in order to collect clinical and therapeutic information on patients aged 65 or older acutely admitted to $>100$ internal medicine and geriatric wards in Italy. More details are available elsewhere [6]. For this analysis, we included all patients enrolled in REPOSI from 2010 to 2017 and prescribed with at least one antidepressant. Prescription appropriateness was assessed according to the American Geriatrics Society Beers Criteria (version 2015) and to the criteria developed in Europe [4,5].

We divided antidepressants in groups and considered them inappropriately prescribed, because 1) they should be always avoided in older people (e.g. TCA, paroxetine in 2017, fluoxetine from 2014), 2) prescribed to patients with a Glomerular Filtration Rate (GFR) $<30 \mathrm{ml} / \mathrm{min}$ (duloxetine), 3) prescribed to patients with a history of falls, 4) co-prescribed with at least other two additional drugs active on the central nervous system (CNS). Furthermore, for the first 3 mostly prescribed SSRIs (sertraline, paroxetine, escitalopram) we also assessed the most clinically relevant potential drug-drug interactions (DDIs) with antithrombotic agents, nonsteroidal anti-inflammatory drugs,fluorochinolone and macrolide antibiotics and amiodarone.

All in all, 4681 were eligible for the analysis. At hospital discharge, 616 patients $(13.2 \%$, 95\% CI: $12.2 \%-14.2 \%)$ were prescribed antidepressants, 39 taking two of them and 2 even three. These were mostly females $(64.8 \%)$, aged 75 years or more (75\%), living in Northern Italy (67.4\%), living with family members $(59.4 \%)$, taking several concomitant drugs $(79.1 \%)$, and being affected by depression $(N=187$, $30.4 \%)$, cerebrovascular diseases $(N=169,27.4 \%)$ and delirium
$(N=143,23.2 \%)$. In the face of a decreasing trend of SSRIs from $64.9 \%$ in 2010 to $52.9 \%$ in 2017 , other antidepressants (SNRI) increased from $30.4 \%$ to $43.4 \%$. The most prescribed class of AD was that of SSRI $(n=361)$, followed by other ADs (i.e. SNRIs; $n=210$ ) and then TCA $(n=33)$. The most prescribed AD (alone or in combination) was sertraline $(N=139,22.6 \%)$, followed by trazodone $(N=130,21.1 \%)$, paroxetine $(N=89,14.5 \%)$ and escitalopram $(N=65,10.5 \%)$. Table 1 shows the profiles of prescription inappropriateness in $\mathrm{AD}$ users. At hospital discharge, 158 patients (26.9\%, 95\% CI: $22.4 \%-29.2 \%)$ were inappropriately prescribed: $39.9 \%$ of them $(N=63)$ prescribed with $\mathrm{AD}$ in combination with other CNS - active drugs, 31.6\% $(n=50)$ with $\mathrm{AD}$ always to be avoided in the elderly and $25.3 \%(n=40)$ with a history of falls. Among patients prescribed with sertraline, alone or in combination, $103(/ 139,74.1 \%)$ had at least a potential DDI: the antithrombotic agents were the most co-prescribed drugs at risk of DDI $(N=96)$, followed by antibiotics $(N=15)$. The same pattern of potential DDI risk was found for paroxetine $(N=66 / 89,74.2 \%$ patients with at least one DDI) and escitalopram $(N=51 / 65,78.5 \%)$.

At hospital admission, 564 patients (12.0\%, 95\% CI: 11.1\%-13.0\%) were prescribed with $\mathrm{ADs}$, being 140 patients $(24.8 \%$, 95\% CI: $21.4 \%-28.6 \%$ ) inappropriately prescribed (Table 1). Also at admission the majority of patients $(N=52,37.1 \%)$ were inappropriately co-prescribed with at least two other CNS drugs, but in this instance there were more patients prescribed with $\mathrm{AD}$ always to be avoided in the elderly.

Among 564 patients prescribed ADs at hospital admission, 88 (15.6\%, 95\% CI: $12.8 \%-18.8 \%)$ stopped them at discharge, while 140 / 4117 patients (3.4\%, 95\%CI: $2.9 \%-4.0 \%$ ) were first prescribed at discharge. Of the latter, $26(18.6 \%)$ were inappropriately prescribed. Among 476 patients prescribed both at admission and discharge, 104/ 121 (85.9\%, 95\%CI: 78.6\% - 91.0\%) continued to be inappropriately prescribed and 327/355 (92.1\%, 95\% CI: $88.8 \%$ - 94.5\%) were still appropriately prescribed from admission to discharge.

In conclusion, this study shows an overall appropriate use of ADs in acutely hospitalized older people in internal medicine and geriatric wards from 2010 to 2017, but still some critical aspects were highlighted: an increasing number of patients prescribed ADs despite their history of falls, the frequent combinations between ADs and drugs acting on CNS and the concomitant use of other drugs that can cause potential drug-drug interactions. The hospital setting should represent an important opportunity to improve the quality of drug prescription for frail, multimorbid and polytreated older patients. In our previous studies we pointed out that hospitalization did fail in this goal [7-10]. With reference to ADs, even if the overall use was often mainly appropriate, physicians should improve their knowledge on the risks related to potential DDI and on the concomitant use of drugs that may increase the risk of falls in the elderly, in order to avoid harmful adverse events for patients and unnecessary direct and indirect costs for National Health Service. 
Table 1

Patients inappropriately prescribed with antidepressants.

\begin{tabular}{|c|c|c|c|c|}
\hline & \multicolumn{2}{|l|}{ Hospital discharge } & \multicolumn{2}{|l|}{ Hospital admission } \\
\hline & Antidepressant users & N (\%) & Antidepressant users & N (\%) \\
\hline & & 616 & & 564 \\
\hline Not appropriate & & $158(26.9)$ & & $140(24.8)$ \\
\hline Always & & $50(31.6)$ & & $52(37.1)$ \\
\hline Amitriptyline & 24 & & 25 & \\
\hline Amoxapine & 0 & & 0 & \\
\hline Clomipramine & 4 & & 4 & \\
\hline Desipramine & 0 & & 0 & \\
\hline Doxepin $>6 \mathrm{mg} / \mathrm{d}$ & 0 & & 0 & \\
\hline Imipramine & 1 & & 2 & \\
\hline Nortriptyline & 2 & & 2 & \\
\hline Protriptyline & 0 & & 0 & \\
\hline Trimipramine & 0 & & 0 & \\
\hline Paroxetine* & 15 & & 13 & \\
\hline Fluoxetine $^{\#}$ & 4 & & 3 & \\
\hline Tranylcypromine & 0 & & 0 & \\
\hline Amitriptyline and psycholeptics & 0 & & 3 & \\
\hline Fluoxetine and psycholeptics & 0 & & 0 & \\
\hline If $\mathrm{GFR}<30 \mathrm{ml} / \mathrm{min}$ & & $5(3.2)$ & & $4(2.9)$ \\
\hline Duloxetine & 5 & & 4 & \\
\hline If co-prescribed with $\geq 2$ CNS active-drugs ${ }^{* *}$ & & $63(39.9)$ & & $52(37.1)$ \\
\hline If there are previous falls and fractures & & $40(25.3)$ & & 32 (22.9) \\
\hline NOT ASSESSABLE & & 1 & & 1 \\
\hline
\end{tabular}

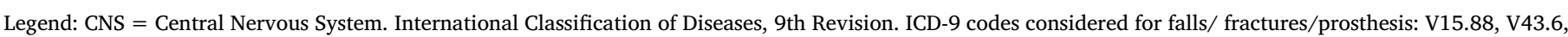
800-829.

* We considered it inappropriately prescribed only in REPOSI 2017 since it has been included in the Beers 2015 version.

\# We considered it inappropriately prescribed from REPOSI 2014.

** Antipsychotics (Anatomical Therapeutic Chemical classification system -ATC: N05A), benzodiazepines, nonbenzodiazepine, benzodiazepine receptor agonist

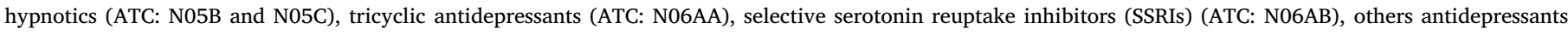
(ATC: N06AX) and opiods (ATC: N02A).

\section{Declaration of Competing Interest}

The authors declare that they have no conflicts of interest.

\section{References}

[1] Parabiaghi A, Franchi C, Tettamanti M, Barbato A, D'Avanzo B, Fortino I, et al. Antidepressants utilization among elderly in Lombardy from 2000 to 2007: dispensing trends and appropriateness. Eur J Clin Pharmacol 2011 Oct;67(10):1077-83.

[2] Boehlen FH, Freigofas J, Herzog W, Meid AD, Saum KU, Schoettker B, et al. Evidence for underuse and overuse of antidepressants in older adults: results of a large population-based study. Int J Geriatr Psychiatry 2019 Apr;34(4):539-47.

[3] American Geriatrics Society 2015 Beers Criteria Update Expert Panel. American Geriatrics Society 2015 updated beers criteria for potentially inappropriate medication use in older adults. J Am Geriatr Soc 2015 Nov;63(11):2227-46. https://doi. org/10.1111/jgs.13702.

[4] Holt S, Schmiedl S, Thürmann PA. Potentially inappropriate medications in the elderly: the PRISCUS list. Dtsch Arztebl Int 2010;107(31-32):543-51.

[5] Renom-Guiteras A, Meyer G, Thürmann PA. The EU(7)-PIM list: a list of potentially inappropriate medications for older people consented by experts from seven European countries. Eur J Clin Pharmacol 2015 Jul;71(7):861-75.

[6] Marcucci M, Franchi C, Nobili A, Mannucci PM, Ardoino I, REPOSI Investigators. Defining aging phenotypes and related outcomes: clues to recognize frailty in hospitalized older patients. J Gerontol A Biol Sci Med Sci 2017 Mar 1;72(3):395-402.

[7] Ardoino I, Rossio R, Di Blanca D, Nobili A, Pasina L, Mannucci PM, et al. Appropriateness of antiplatelet therapy for primary and secondary cardio- and cerebrovascular prevention in acutely hospitalized older people. $\mathrm{Br} \mathrm{J}$ Clin Pharmacol 2017 Nov;83(11):2528-40. https://doi.org/10.1111/bcp.13355.

[8] Franchi C, Antoniazzi S, Proietti M, Nobili A, Mannucci PM. SIM-AF Collaborators. Appropriateness of oral anticoagulant therapy prescription and its associated factors in hospitalized older people with atrial fibrillation. Br J Clin Pharmacol 2018 Sep;84(9):2010-9.

[9] Franchi C, Tettamanti M, Djade CD, Pasina L, Mannucci PM, Onder G, et al, Elearning in order to improve drug prescription for hospitalized older patients: a cluster-randomized controlled study. Br J Clin Pharmacol 2016 Jul;82(1):53-63.

[10] Franchi C, Rossio R, Ardoino I, Mannucci PM, Nobili A. REPOSI Collaborators. Inappropriate prescription of benzodiazepines in acutely hospitalized older patients. Eur Neuropsychopharmacol 2019 Jul;29(7):871-9. https://doi.org/10. 1016/j.euroneuro.2019.05.004.
Franchi Carlotta ${ }^{\text {cy,*, }}$ Rossio Raffaella ${ }^{\mathrm{cz}}$, Ardoino Ilaria ${ }^{\mathrm{cy}}$, Nobili Alessandro $^{\text {cy }}$, Mannucci Pier Mannuccio ${ }^{\text {da }}$, REPOSI Collaborators (Steering Committee, Pier Mannuccio Mannucci ${ }^{\mathrm{a}}$, Alessandro Nobili ${ }^{\mathrm{b}}$, Antonello Pietrangelo ${ }^{c}$, Francesco Perticone ${ }^{c}$, Giuseppe Licata ${ }^{c}$, Francesco Violi ${ }^{\mathrm{c}}$, Gino Roberto Corazza ${ }^{\mathrm{d}}$, Salvatore Corrao ${ }^{\mathrm{e}}$, Alessandra Marengoni ${ }^{\mathrm{f}}$, Francesco Salerno ${ }^{\mathrm{g}}$, Matteo Cesari ${ }^{\mathrm{h}}$, Mauro Tettamanti ${ }^{\mathrm{i}}$, Luca Pasina ${ }^{\mathrm{i}}$, Carlotta Franchi ${ }^{\mathrm{i}}$ ) (Clinical Data Monitoring and Revision, Carlotta Franchi ${ }^{\mathrm{j}}$, Laura Cortesi, Mauro Tettamanti ${ }^{j}$, Gabriella Miglio ${ }^{j}$ ) (Database Management and Statistics, Mauro Tettamanti ${ }^{\mathrm{k}}$, Laura Cortesi ${ }^{\mathrm{k}}$, Ilaria Ardoino ${ }^{\mathrm{k}}$, Alessio Novella ${ }^{k}$ ) (Investigators, Domenico Prisco ${ }^{1}$, Elena Silvestri, Giacomo Emmi ${ }^{1}$, Alessandra Bettiol ${ }^{1}$, Cenci Caterina ${ }^{1}$, Gianni Biolo ${ }^{\mathrm{m}}$, Michela Zanetti $^{\mathrm{m}}$, Martina Guadagni ${ }^{\mathrm{m}}$, Michele Zaccari ${ }^{\mathrm{m}}$, Massimiliano Chiuch ${ }^{\mathrm{m}}$, Michele Zaccari ${ }^{\mathrm{m}}$, Massimo Vanoli ${ }^{\mathrm{n}}$,

Giulia Grignani ${ }^{\mathrm{n}}$, Edoardo Alessandro Pulixi ${ }^{\mathrm{n}}$, Mauro Bernardi ${ }^{\circ}$, Silvia Li Bassi ${ }^{\circ}$, Luca Santi ${ }^{\circ}$, Giacomo Zaccherini $^{\circ}$, Graziana Lupattelli ${ }^{\mathrm{P}}$, Elmo Mannarino ${ }^{\mathrm{p}}$, Vanessa Bianconi ${ }^{\mathrm{p}}$, Francesco Paciullo ${ }^{\mathrm{p}}$, Riccardo Alcidi ${ }^{\mathrm{p}}$, Ranuccio Nuti ${ }^{\mathrm{q}}$, Roberto Valenti ${ }^{\mathrm{q}}$, Martina Ruvio ${ }^{\mathrm{q}}$, Silvia Cappelli ${ }^{\mathrm{q}}$, Alberto Palazzuoli ${ }^{\mathrm{q}}$, Domenico Girelli ${ }^{\mathrm{r}}$, Fabiana Busti ${ }^{\mathrm{r}}$, Giacomo Marchi ${ }^{\mathrm{r}}$, Mario Barbagallo ${ }^{\mathrm{s}}$, Ligia Dominguez,

Floriana Cocita ${ }^{s}$, Vincenza Beneduce ${ }^{s}$, Lidia Plances ${ }^{s}$, Salvatore Corrao ${ }^{t}$, Giuseppe Natoli ${ }^{t}$, Salvatore Mularo ${ }^{t}$, Massimo Raspanti,

Federica Cavallaro ${ }^{\mathrm{t}}$, Marco Zoli ${ }^{\mathrm{u}}$, Ilaria Lazzari ${ }^{\mathrm{u}}$, Mattia Brunori ${ }^{\mathrm{u}}$, Elisa Fabbri ${ }^{u}$, Donatella Magalotti ${ }^{u}$, Raffaella Arnò ${ }^{u}$, Franco Laghi Pasini ${ }^{\mathrm{v}}$, Pier Leopoldo Capecchi ${ }^{\mathrm{v}}$, Giuseppe Palascianow, Maria Ester Modeo $^{w}$, Carla Di Gennarow ${ }^{w}$, Maria Domenica Cappellini ${ }^{\mathrm{x}}$, Diletta Maira ${ }^{\mathrm{x}}$, Valeria Di Stefano ${ }^{\mathrm{x}}$, Giovanna Fabio ${ }^{\mathrm{x}}$, Sonia Seghezzi ${ }^{\mathrm{x}}$, Marta Mancarella ${ }^{\mathrm{x}}$, Margherita Migone De Amicis ${ }^{\mathrm{x}}$, Giacomo De Luca ${ }^{\mathrm{x}}$, Natalia Scaramellini ${ }^{\mathrm{x}}$, Matteo Cesari ${ }^{\mathrm{y}}$, Paolo Dionigi Rossi ${ }^{\mathrm{y}}$,

Sarah Damanti ${ }^{\mathrm{y}}$, Marta Clerici ${ }^{\mathrm{y}}$, Federica Conti ${ }^{\mathrm{y}}$, Giulia Bonini ${ }^{\mathrm{y}}$, Barbara Brignolo Ottolini ${ }^{\mathrm{y}}$, Antonio Di Sabatino ${ }^{\mathrm{z}}$, Emanuela Miceli ${ }^{\mathrm{z}}$, Marco Vincenzo Lenti ${ }^{\mathrm{z}}$, Martina Pisati ${ }^{\mathrm{z}}$, Costanza Caccia Dominioni ${ }^{\mathrm{z}}$, Giovanni Murialdo $^{\text {aa }}$, Alessio Marra ${ }^{\text {aa }}$, Federico Cattaneo ${ }^{\text {aa }}$, Roberto Pontremoli ${ }^{\text {aa }}$, Valentina Beccati ${ }^{\text {aa }}$, Giulia Nobili ${ }^{\text {aa }}$, 
Maria Beatrice Secchi ${ }^{\mathrm{ab}}$, Davide Ghelfi ${ }^{\mathrm{ab}}$, Luigi Anastasio ${ }^{\mathrm{ac}}$, Lucia Sofia $^{\text {ac }}$, Maria Carbone ${ }^{\text {ac }}$, Francesco Cipollone ${ }^{\text {ad }}$, Maria Teresa Guagnano ${ }^{\text {ad }}$, Emanuele Valeriani ${ }^{\text {ad }}$, Ilaria Rossi ${ }^{\text {ad }}$, Gerardo Mancuso $^{\text {ae }}$, Daniela Calipari ${ }^{\mathrm{ae}}$, Mosè Bartone ${ }^{\mathrm{ae}}$, Giuseppe Delitala ${ }^{\text {af }}$, Maria Berria ${ }^{\text {af }}$, Chiara Pes ${ }^{\text {af }}$, Alessandro Delitala ${ }^{\text {af }}$, Maurizio Muscaritoli $^{\text {ag }}$, Alessio Molfino ${ }^{\text {ag }}$, Enrico Petrillo ${ }^{\text {ag, }}$, Giuseppe Zuccalà $^{\text {ah }}$, Gabriella D'Aurizio ${ }^{\text {ah }}$, Giuseppe Romanelli ${ }^{\text {ai }}$, Alessandra Marengoni ${ }^{\text {ai }}$, Alberto Zucchelli ${ }^{\text {ai }}$, Francesca Manzoni ${ }^{\text {ai }}$, Andrea Volpini $^{\text {ai }}$, Antonio Picardi ${ }^{\text {aj }}$, Umberto Vespasiani Gentilucci ${ }^{\text {aj, }}$ Paolo Gallo ${ }^{\text {aj }}$, Chiara Dell'Unto ${ }^{\text {aj }}$, Giorgio Annoni ${ }^{\text {ak }}$, Maurizio Corsi ${ }^{\text {ak }}$, Giuseppe Bellelli $^{\text {ak }}$, Sara Zazzetta ${ }^{\text {ak }}$, Paolo Mazzola ${ }^{\text {ak }}$, Hajnalka Szabo ${ }^{\text {ak }}$, Alessandra Bonfanti ${ }^{\text {ak }}$, Franco Arturi ${ }^{\mathrm{al}}$, Elena Succurro ${ }^{\text {al }}$, Mariangela Rubino ${ }^{\text {al }}$, Bruno Tassone ${ }^{\text {al }}$, Giorgio Sesti ${ }^{\text {al, }}$ Medicina Interna, Maria Grazia Serra ${ }^{\mathrm{am}}$, Maria Antonietta Bleve ${ }^{\mathrm{am}}$, Laura Gasbarrone $^{\text {an }}$, Maria Rosaria Sajeva ${ }^{\text {an }}$, Antonio Brucato ${ }^{\text {ao, }}$ Silvia Ghidoni ${ }^{\text {ao }}$, Fabrizio Fabris ${ }^{\text {ap }}$, Irene Bertozzi ${ }^{\text {ap }}$, Giulia Bogoni ${ }^{\text {ap }}$,

Maria Victoria Rabuini $^{\text {ap }}$, Elisabetta Cosi ${ }^{\text {ap }}$, Paolo Scarinzi ${ }^{\text {ap }}$, Annalisa Amabile $^{\text {ap }}$, Elisabetta Omenetto ${ }^{\text {ap }}$, Tancredi Prandini ${ }^{\text {ap }}$, Roberto Manfredini $^{\text {aq }}$, Fabio Fabbian ${ }^{\text {aq }}$, Benedetta Boari ${ }^{\text {aq }}$, Alfredo De Giorgi ${ }^{\text {aq }}$, Ruana Tiseo ${ }^{\text {aq }}$, Roberto De Giorgio ${ }^{\text {aq }}$,

Giuseppe Paolisso $^{\text {ar }}$, Maria Rosaria Rizzo ${ }^{\text {ar }}$, Claudio Borghi ${ }^{\text {as }}$, Enrico Strocchi ${ }^{\text {as }}$, Eugenia Ianniello ${ }^{\text {as }}$, Mario Soldatias ${ }^{\text {as }}$, Carlo Sabbà ${ }^{\text {at, }}$ Francesco Saverio Vella ${ }^{\text {at }}$, Patrizia Suppressa ${ }^{\text {at }}$, Andrea Schilardi ${ }^{\text {at }}$, Francesca Loparco $^{\text {at }}$, Giovanni Michele De Vincenzo ${ }^{\text {at }}$, Alessio Comitangelo ${ }^{\text {at }}$, Emanuele Amoruso ${ }^{\text {at }}$, Luigi Fenoglio au, Andrea Falcetta $^{\text {au }}$, Christian Bracco ${ }^{\text {au }}$, Anna L. Fracanzani ${ }^{\text {av }}$, Silvia Fargion $^{\text {av }}$, Silvia Tiraboschi ${ }^{\text {av }}$, Annalisa Cespiati ${ }^{\text {av }}$, Giovanna Oberti $^{\text {av }}$, Giordano Sigon ${ }^{\text {av }}$, Flora Peyvandiaw, Raffaella Rossio $^{\text {aw }}$, Barbara Ferrari ${ }^{\text {aw }}$, Giulia Colombo ${ }^{\text {aw }}$, Pasquale Agosti $^{\text {aw }}$, Valter Monzani ${ }^{\text {ax }}$, Valeria Savojardo ${ }^{\text {ax }}$, Christian Folli $^{\text {ax }}$, Giuliana Ceriani ${ }^{\mathrm{ax}}$, Francesco Salerno ${ }^{\mathrm{ay}}$, Giada Pallini $^{\mathrm{ay}}$, Franco Dallegria $^{\mathrm{az}}$, Luciano Ottonello ${ }^{\mathrm{az}}$, Luca Liberale ${ }^{\mathrm{az}}$, Lara Caserza $^{\mathrm{az}}$, Kassem Salam ${ }^{\mathrm{az}}$, Nicola Lucio Liberato ${ }^{\mathrm{ba}}$,

Tiziana Tognin $^{\text {ba }}$, Giovanni Battista Bianchi ${ }^{\text {bb }}$, Sabrina Giaquinto ${ }^{\text {bb }}$, Francesco Purrello $^{\mathrm{bc}}$, Antonino Di Pino ${ }^{\mathrm{bc}}$, Salvatore Piro ${ }^{\mathrm{bc}}$, Renzo Rozzini ${ }^{\text {bd }}$, Lina Falanga ${ }^{\text {bd }}$, Elena Spazzini ${ }^{\text {bd, }}$,

Camillo Ferrandina $^{\text {bd }}$, Giuseppe Montrucchio ${ }^{\text {be }}$, Paolo Petitti ${ }^{\text {be }}$, Paolo Peasso ${ }^{\text {be }}$, Edoardo Favale ${ }^{\text {be }}$, Cesare Poletto ${ }^{\text {be }}$, Raffaella Salmi ${ }^{\text {bf }}$, Piergiorgio Gaudenzi ${ }^{\text {bf }}$, Francesco Violi ${ }^{\text {bg }}$, Ludovica Perri ${ }^{\text {bg }}$, Raffaele Landolfi $^{\text {bh }}$, Massimo Montalto ${ }^{\text {bh }}$, Antonio Mirijello ${ }^{\text {bh }}$, Luigina Guasti $^{\text {bi }}$, Luana Castiglioni ${ }^{\text {bi }}$, Andrea Maresca ${ }^{\text {bi }}$, Alessandro Squizzato ${ }^{\text {bi }}$, Leonardo Campiotti ${ }^{\mathrm{bi}}$, Alessandra Grossi ${ }^{\mathrm{bi}}$, Marco Bertolotti ${ }^{\mathrm{bj}}$, Chiara Mussi ${ }^{\mathrm{bj}}$, Giulia Lancellotti ${ }^{\mathrm{bj}}$, Maria Vittoria Libbra ${ }^{\text {bj }}$, Giulia Dondi ${ }^{\text {bj }}$, Elisa Pellegrini ${ }^{\text {bj }}$, Lucia Carulli ${ }^{\text {bj }}$, Matteo Galassi ${ }^{\mathrm{bj}}$, Yasmine Grassi ${ }^{\mathrm{bj}}$, Francesco Perticone ${ }^{\mathrm{bk}}$, Maria Perticone $^{\mathrm{bk}}$, Rosa Battaglia ${ }^{\mathrm{bk}}$, Marco FIlice ${ }^{\mathrm{bk}}$, Raffaele Maio ${ }^{\mathrm{bk}}$, Vincenzo Stanghellini ${ }^{\mathrm{bl}}$, Eugenio Ruggeri ${ }^{\mathrm{bl}}$, Sara del Vecchio ${ }^{\mathrm{bl}}$, Andrea Salvi $^{\mathrm{bm}}$, Roberto Leonardi ${ }^{\mathrm{bm}}$, Giampaolo Damiani ${ }^{\mathrm{bm}}$, William Capeci ${ }^{\text {bn }}$, Armando Gabrielli ${ }^{\text {bn }}$, Massimo Mattioli ${ }^{\text {bn }}$, Giuseppe Pio Martino ${ }^{\mathrm{bn}}$, Lorenzo Biondi ${ }^{\mathrm{bn}}$, Pietro Pettinari ${ }^{\mathrm{bn}}$, Riccardo Ghio $^{\text {bo }}$, Anna Dal Col ${ }^{\text {bo }}$, Salvatore Minisola ${ }^{\text {bp }}$, Luciano Colangelo $^{\text {bp }}$, Mirella Cilli ${ }^{\text {bp }}$, Giancarlo Labbadia ${ }^{\text {bp }}$, Antonella Afeltra $^{\text {bq }}$, Benedetta Marigliano ${ }^{\text {bq }}$, Maria Elena Pipita ${ }^{\text {bq }}$, Pietro Castellino ${ }^{\text {br }}$, Luca Zanoli ${ }^{\text {br }}$, Samuele Pignataro ${ }^{\text {br }}$, Alfio Gennaro ${ }^{\text {br }}$, Julien Blanco $^{\text {br }}$, Valter Saracco ${ }^{\text {bs }}$, Marisa Fogliati ${ }^{\text {bs }}$, Carlo Bussolino ${ }^{\text {bs }}$, Francesca Mete $^{\text {bt }}$, Miriam Gino ${ }^{\text {bt }}$, Antonio Cittadini ${ }^{\text {bu }}$, Carlo Vigorito ${ }^{\text {bu }}$, Michele Arcopinto ${ }^{\text {bu }}$, Andrea Salzano ${ }^{\text {bu }}$, Emanuele Bobbio ${ }^{\text {bu }}$, Alberto Maria Marra ${ }^{\text {bu }}$, Domenico Sirico ${ }^{\text {bu }}$, Guido Moreo ${ }^{\text {bv }}$, Francesca Gasparini ${ }^{\text {bv }}$, Silvia Prolo ${ }^{\text {bv }}$, Gloria Pina ${ }^{\text {bv }}$,

Alberto Ballestrero $^{\text {bw }}$, Fabio Ferrando ${ }^{\text {bw }}$, Sergio Berra ${ }^{\text {bx }}$, Simonetta Dassi ${ }^{\text {bx }}$, Maria Cristina Nava ${ }^{\text {bx }}$, Bruno Graziella ${ }^{\text {by }}$, Stefano Baldassarre ${ }^{\text {by }}$, Salvatore Fragapani ${ }^{\text {by }}$, Gabriella Gruden ${ }^{\text {by }}$, Giorgio Galanti ${ }^{\text {bz }}$, Gabriele Mascherini ${ }^{\mathrm{bz}}$, Cristian Petri ${ }^{\text {bz }}$, Laura Stefani $^{\text {bz }}$, Margherita Girino ${ }^{\text {ca }}$, Valeria Piccinelli ${ }^{\text {ca }}$, Francesco Nasso $^{\mathrm{cb}}$, Vincenza Gioffrè ${ }^{\mathrm{cb}}$, Maria Pasquale ${ }^{\mathrm{cb}}$, Giuseppe Scattolin ${ }^{\mathrm{cc}}$, Sergio Martinellic ${ }^{\mathrm{cc}}$, Mauro Turrin ${ }^{\mathrm{cc}}$,
Leonardo Sechi ${ }^{\text {cd }}$, Cristina Catena ${ }^{\text {cd }}$, Gianluca Colussi ${ }^{\text {cd }}$, Nicola Passariello ${ }^{\mathrm{ce}}$, Luca Rinaldi ${ }^{\mathrm{ce}}$, Franco Berti ${ }^{\mathrm{cf}}$, Giuseppe Famularo ${ }^{\mathrm{cf}}$, Patrizia Tarsitani ${ }^{\mathrm{cf}}$, Roberto Castello ${ }^{\mathrm{cg}}$, Michela Pasino $^{\text {cg }}$, Gian Paolo Ceda ${ }^{\text {ch }}$, Marcello Giuseppe Maggio ${ }^{\text {ch }}$, Simonetta Morganti ${ }^{\text {ch }}$, Andrea Artoni ${ }^{\text {ch }}$, Stefano Del Giacco ${ }^{\text {ci }}$, Davide Firinu $^{\text {ci }}$, Francesca Losa ${ }^{\text {ci }}$, Giovanni Paoletti ${ }^{\text {ci }}$, Giulia Costanzo ${ }^{\text {ci }}$, Giuseppe Montalto $^{\mathrm{cj}}$, Anna Licata ${ }^{\mathrm{cj}}$, Valentina Malerba ${ }^{\mathrm{cj}}$, Filippo Alessandro Montalto ${ }^{\mathrm{cj}}$, Antonino Lasco ${ }^{\mathrm{ck}}$, Giorgio Basile ${ }^{\mathrm{ck}}$, Antonino Catalano $^{\mathrm{ck}}$, Lorenzo Malatino ${ }^{\mathrm{cl}}$, Benedetta Stancanelli ${ }^{\mathrm{cl}}$, Valentina Terranova ${ }^{\mathrm{cl}}$, Salvatore Di Marca ${ }^{\mathrm{cl}}$, Rosario Di Quattro ${ }^{\mathrm{cl}}$, Lara La Malfa $^{\mathrm{cl}}$, Rossella Caruso ${ }^{\mathrm{cl}}$, Patrizia Mecocci ${ }^{\mathrm{cm}}$, Carmelinda Ruggiero $^{\mathrm{cm}}$, Virginia Boccardi ${ }^{\mathrm{cm}}$, Tiziana Meschi ${ }^{\mathrm{cn}}$, Fulvio Lauretani ${ }^{\mathrm{cn}}$, Andrea Ticinesi ${ }^{\mathrm{cn}}$, Antonio Nouvenne ${ }^{\mathrm{cn}}$, Pietro Minuz ${ }^{\mathrm{co}}$, Luigi Fondrieschi ${ }^{\mathrm{co}}$, Mario Pirisi ${ }^{\mathrm{cp}}$, Gian Paolo $\mathrm{Fra}^{\mathrm{cp}}$,

Daniele Sola $^{\mathrm{cp}}$, Massimo Porta ${ }^{\mathrm{cq}}$, Piero Riva ${ }^{\mathrm{cq}}$, Roberto Quadri ${ }^{\mathrm{cr}}$, Erica Larovere ${ }^{\text {cr }}$, Marco Novelli ${ }^{\text {cr }}$, Giorgio Scanzi ${ }^{\text {cs }}$, Caterina Mengoli ${ }^{\text {cs }}$, Stella Provini ${ }^{\mathrm{cs}}$, Laura Ricevuti ${ }^{\mathrm{cs}}$, Emilio Simeone ${ }^{\mathrm{ct}}$, Rosa Scurti ${ }^{\mathrm{ct}}$, Fabio Tolloso ${ }^{\text {ct }}$, Roberto Tarquini ${ }^{\mathrm{cu}}$, Alice Valoriani ${ }^{\mathrm{cu}}$, Silvia Dolenti ${ }^{\mathrm{cu}}$, Giulia Vannini $^{\mathrm{cu}}$, Alberto Tedeschi ${ }^{\mathrm{cv}}$, Lucia Trotta ${ }^{\mathrm{cv}}$, Riccardo Volpi ${ }^{\mathrm{cw}}$, Pietro Bocchi ${ }^{\mathrm{cw}}$, Alessandro Vignali ${ }^{\mathrm{cw}}$, Sergio Harari ${ }^{\mathrm{cx}}$, Chiara Lonati ${ }^{\mathrm{cx}}$, Mara Cattaneo $^{\mathrm{cx}}$, Federico Napoli ${ }^{\mathrm{cx}}$ ),

${ }^{\mathrm{cy}}$ Department of Neuroscience, Istituto di Ricerche Farmacologiche Mario Negri IRCCS, Milano, Italy

${ }^{\mathrm{cz}}$ Department of Pathophysiology and Transplantation, Fondazione IRCCS Cà Granda Ospedale Maggiore Policlinico, Milan, Italy

${ }^{\text {da }}$ Scientific Direction, Fondazione IRCCS Ca' Granda Ospedale Maggiore Policlinico, Milano, Italy

a Fondazione IRCCS Cà Granda Ospedale Maggiore Policlinico, Milano, Italy

${ }^{\mathrm{b}}$ Istituto di Ricerche Farmacologiche Mario Negri IRCCS, Milano, Italy ${ }^{\mathrm{c}}$ Policlinico Umberto I, Roma, Prima Clinica Medica, Italy

${ }^{\mathrm{d}}$ Reparto 11, IRCCS Policlinico San Matteo di Pavia, Pavia, Clinica Medica I, Italy

e ARNAS Civico, Di Cristina, Benfratelli, DiBiMIS, Università di Palermo, Palermo, Italy

${ }^{\mathrm{f}}$ Spedali Civili di Brescia, Brescia, Italy

${ }^{g}$ IRCCS Policlinico San Donato Milanese, Milano, Italy

${ }^{\mathrm{h}}$ UO Geriatria, Università degli Studi di, Milano, Italy

${ }^{\mathrm{i}}$ Istituto di Ricerche Farmacologiche Mario Negri IRCCS, Milano, Italy

${ }^{\mathrm{j}}$ Istituto di Ricerche Farmacologiche Mario Negri IRCCS, Milano, Italy

${ }^{\mathrm{k}}$ Istituto di Ricerche Farmacologiche Mario Negri IRCCS, Milano, Italy

${ }^{1}$ Azienda Ospedaliero Universitaria Careggi Firenze, Medicina Interna Interdisciplinare, Italy

${ }^{\mathrm{m}}$ Azienda Sanitaria Universitaria Integrata di Trieste, Clinica Medica Generale e Terapia Medica, Italy

${ }^{\mathrm{n}}$ Azienda Ospedaliera della Provincia di Lecco, Ospedale di Merate, Lecco, Medicina Interna, Italy

${ }^{\circ}$ Azienda Ospedaliera Policlinico Sant'Orsola-Malpighi, Bologna, Semeiotica Medica Bernardi, Italy

${ }^{\mathrm{P}}$ Azienda Ospedaliera Santa Maria della Misericordia, Perugia, Medicina Interna, Italy

${ }^{\mathrm{q}}$ Azienda Ospedaliera Università Senese, Siena, Medicina Interna I, Italy ${ }^{\mathrm{r}}$ Azienda Ospedaliera Universitaria Integrata di Verona, Verona, Medicina Generale e Malattie Aterotrombotiche e Degenerative, Italy ${ }^{\mathrm{s}}$ Azienda Ospedaliera Universitaria Policlinico Giaccone Policlinico di Palermo, Palermo, Unità Operativa di Geriatria e Lungodegenza, Italy ${ }^{\mathrm{t}}$ A.R.N.A.S. Civico, Di Cristina, Benfratelli, Palermo, UOC Medicina Interna ad Indirizzo Geriatrico-Riabilitativo, Italy

${ }^{u}$ Azienda Ospedaliera Universitaria Policlinico S. Orsola-Malpighi, Bologna, Unità Operativa di Medicina Interna, Italy

${ }^{\mathrm{v}}$ Azienda Ospedaliera Universitaria Senese, Siena, Unità Operativa Complessa Medicina 2, Italy

${ }^{\mathrm{w}}$ Azienda Ospedaliero-Universitaria Consorziale Policlinico di Bari, Bari, Medicina Interna Ospedaliera "L. D'Agostino", Medicina Interna Universitaria "A. Murri", Italy

${ }^{\mathrm{x}}$ Fondazione IRCCS Cà Granda Ospedale Maggiore Policlinico, Milano, 
Unità Operativa Medicina Interna IA, Italy

${ }^{\mathrm{y}}$ Fondazione IRCCS Cà Granda Ospedale Maggiore Policlinico, Milano, Geriatria, Italy

${ }^{\mathrm{z}}$ IRCCS Policlinico San Matteo di Pavia, Pavia, Clinica Medica I, Reparto 11, Italy

${ }^{\text {aa }}$ IRCCS Azienda Ospedaliera Universitaria San Martino-IST di Genova, Genova, Clinica di Medicina Interna 2, Italy ${ }^{\text {ab }}$ Ospedale Bassini di Cinisello Balsamo, Milano, Divisione Medicina, Italy

${ }^{\text {ac }}$ Ospedale Civile Jazzolino di Vibo Valentia, Vibo Valentia, Medicina interna, Italy

${ }^{\text {ad }}$ Ospedale Clinicizzato SS. Annunziata, Chieti, Clinica Medica, Italy ae Ospedale Giovanni Paolo II Lamezia Terme, Catanzaro, Unità Operativa Complessa Medicina Interna, Italy

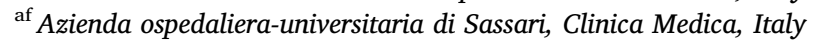
${ }^{\text {ag }}$ Policlinico Umberto I, Sapienza Università di Roma, Medicina Interna e Nutrizione Clinica Policlinico Umberto I, Italy

${ }^{\text {ah }}$ Policlinico Universitario A. Gemelli, Roma, Roma, Unità Operativa Complessa Medicina d'Urgenza e Pronto Soccorso, Italy

${ }^{\text {ai }}$ Spedali Civili di Brescia, Brescia, Geriatria, Italy

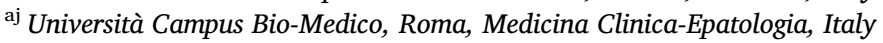

${ }^{\mathrm{ak}}$ Università degli studi di Milano-Bicocca Ospedale S. Gerardo, Monza, Unità Operativa di Geriatria, Italy

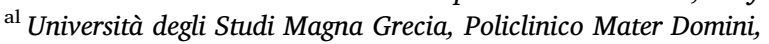
Catanzaro, Unità Operativa Complessa di, Italy

${ }^{\mathrm{am}}$ Azienda Ospedaliera "Cardinale Panico" Tricase, Lecce, Unità Operativa Complessa Medicina, Italy

${ }^{\text {an }}$ Azienda Ospedaliera Ospedale San Camillo Forlanini, Roma, Medicina Interna 1, Italy

${ }^{\text {ao }}$ Azienda Ospedaliera Papa Giovanni XXIII, Bergamo, Medicina 1, Italy

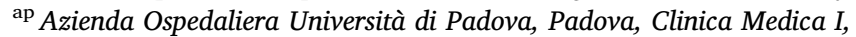
Italy

${ }^{\text {aq }}$ Azienda Ospedaliera - Universitaria Sant'Anna, Ferrara, Unità Operativa Clinica Medica, Italy

${ }^{a r}$ Azienda Ospedaliera Universitaria della Seconda Università degli Studi di Napoli, Napoli, VI Divisione di Medicina Interna e Malattie Nutrizionali dell'Invecchiamento, Italy

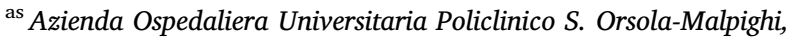
Bologna, Unità Operativa di Medicina Interna Borghi, Italy ${ }^{\text {at }}$ Azienda Ospedaliero-Universitaria Consorziale Policlinico di Bari, Bari, Medicina Interna Universitaria C. Frugoni, Italy

${ }^{\text {au }}$ Azienda Sanitaria Ospedaliera Santa Croce e Carle di Cuneo, Cuneo, S. C. Medicina Interna, Italy

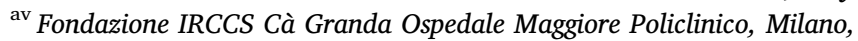
Medicina Interna $1 B$, Italy

${ }^{\mathrm{aw}}$ Fondazione IRCCS Cà Granda Ospedale Maggiore Policlinico, Milano, UOC Medicina generale - Emostasi e trombosi, Italy

${ }^{\mathrm{ax}}$ Fondazione IRCCS Cà Granda Ospedale Maggiore Policlinico, Milano, Medicina Interna Alta Intensità, Italy ay IRCCS Policlinico San Donato e Università di Milano, San, Donato Milanese, Medicina Interna, Italy

${ }^{\mathrm{az}}$ Università di Genova, Genova, Medicina Interna 1, Italy ${ }^{\text {ba }}$ ASST di Pavia, UOSD Medicina Interna, Ospedale di Casorate Primo, Pavia, Italy

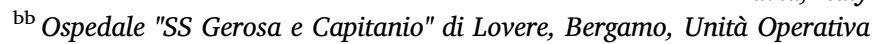
Complessa di Medicina Generale, Azienda Ospedaliera "Bolognini" di Seriate, Bergamo, Italy

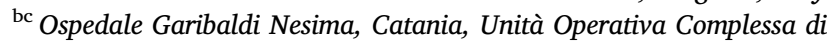
Medicina Interna, Italy

bd Ospedale Poliambulanza, Brescia, Medicina Interna e Geriatria, Italy

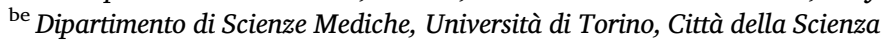
e della Salute, Torino, Medicina Interna 2 U. Indirizzo d'Urgenza, Italy

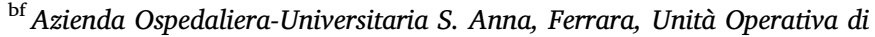
Medicina Ospedaliera II, Italy

${ }^{\mathrm{bg}}$ Policlinico Umberto I, Roma, Prima Clinica Medica, Italy ${ }^{\mathrm{bh}}$ Policlinico Universitario A. Gemelli, Roma, Clinica Medica, Italy

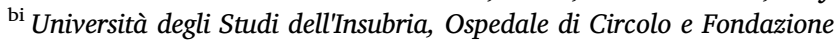

Macchi, Varese, Medicina Interna I, Italy

bj Università di Modena e Reggio Emilia, Azienda Ospedaliero-Universitaria di Modena, Ospedale Civile di Baggiovara, Unità Operativa di Geriatria,

Italy

${ }^{\mathrm{bk}}$ Università Magna Grecia Policlinico Mater Domini, Catanzaro, Unità Operativa Malattie Cardiovascolari Geriatriche, Italy

${ }^{\mathrm{bl}}$ Dipartimento di Scienze Mediche e Chirurgiche, Unità Operativa di Medicina Interna, Università degli Studi di Bologna/Azienda OspedalieroUniversitaria S.Orsola-Malpighi, Bologna, Italy

${ }^{\mathrm{bm}}$ Spedali Civili di Brescia, U.O. 3a Medicina Generale, Italy

${ }^{\text {bn } C l i n i c a}$ Medica, Azienda Ospedaliera Universitaria - Ospedali Riuniti di Ancona, Italy

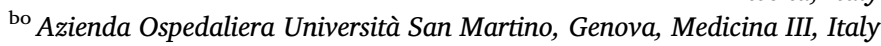

${ }^{\text {bp }}$ Policlinico Umberto I, Roma, SMSCO3 - Medicina Interna A e Malattie Metaboliche dell'osso, Italy

${ }^{\mathrm{bq}}$ Policlinico Campus Biomedico Roma, Roma, Medicina Clinica, Italy

${ }^{\mathrm{br}}$ Azienda Ospedaliera Universitaria Policlinico - V. Emanuele, Catania, Dipartimento di Medicina, Italy

${ }^{\text {bs }}$ Ospedale Cardinal Massaia Asti, Medicina A, Italy

${ }^{\text {bt }}$ Ospedale degli Infermi di Rivoli, Torino, Medicina Interna, Italy

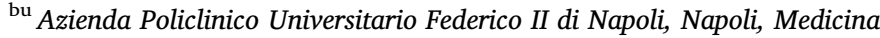
Interna e Riabilitazione Cardiologica, Italy

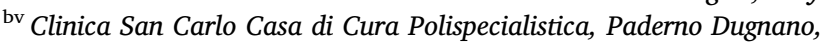
Milano, Unità Operativa di Medicina Interna, Italy

${ }^{\mathrm{bw}}$ Clinica Di Medicina Interna ad Indirizzo Oncologico, Azienda

Ospedaliera Università San Martino di Genova, Italy

${ }^{\mathrm{bx}}$ Medicina Interna, Azienda Ospedaliera Guido Salvini, Garnagnate, Milano, Italy

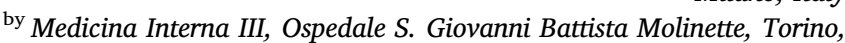
Italy

${ }^{\mathrm{bz}}$ Agenzia di Medicina dello Sport, AOUC Careggi, Firenze, Italy

${ }^{\text {ca } M e d i c i n a ~ I n t e r n a, ~ O s p e d a l e ~ S . ~ S p i r i t o ~ C a s a l e ~ M o n f e r r a t o, ~ A l e s s a n d r i a, ~}$ Italy

${ }^{\mathrm{cb}}$ Struttura Operativa Complessa di Medicina Interna, Ospedale Santa Maria degli Ungheresi, Reggio Calabria, Italy ${ }^{\mathrm{cc}}$ Medicina Interna, Ospedale di Monselice, Padova, Italy

${ }^{\text {cd } C l i n i c a}$ Medica, Azienda Ospedaliera Universitaria, Udine, Italy

${ }^{\text {ce }}$ Presidioe Medico di Marcianise, Napoli, Medicina Interna, Italy

${ }^{\mathrm{cf}}$ Azieneda Ospedaliera San Camillo Forlanini, Roma, Medicina Interna II, Italy

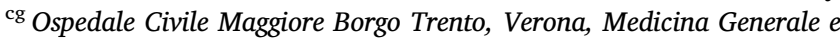
Sezione di Decisione Clinica, Italy ${ }^{\mathrm{ch}}$ Azienda Ospedaliero Universitaria di Parma, U.O.C Clinica Geriatrica, Italy

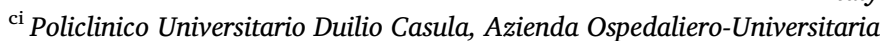
di Cagliari, Cagliari, Medicina Interna, Allergologia ed Immunologia Clinica,

${ }^{\mathrm{cj}}$ Azienda Ospedaliera Universitaria Policlinico Paolo Giaccone, Palermo, UOC di Medicina Interna, Italy

${ }^{\mathrm{ck}}$ Azienda Ospedaliera Universitaria Policlinico G. Martino, Messina, Unità Operativa di Geriatria, Italy

${ }^{\mathrm{cl}}$ Azienda Ospedaliera per l'Emergenza Cannizzaro, Catania, Clinica Medica Università di Catania, Italy

${ }^{\mathrm{cm}}$ Università degli Studi di Perugia-Azienda Ospedaliera S.M. della Misericordia, Perugia, Struttura Complessa di Geriatria, Italy

${ }^{\mathrm{cn}}$ Azienda Ospedaliera Universitaria di Parma, U.O Medicina Interna e Lungodegenza Critica, Italy

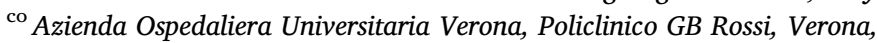
Medicina Generale per lo Studio ed il Trattamento dell'Ipertensione Arteriosa, Italy

${ }^{\mathrm{cp}}$ Azienda Ospedaliera Universitaria Maggiore della Carità, Medicina Interna 1, Italy

${ }^{\mathrm{cq}}$ Azienda Ospedaliera Universitaria Città della Salute e della Scienza di Torino, Medicina Interna $1 U$, Italy

${ }^{\mathrm{cr}}$ Ospedale di Ciriè, ASL TO4, Torino, S.C. Medicina Interna, Italy ${ }^{\mathrm{cs}}$ ASST Lodi, Presidio di Codogno, Medicina, Milano, Italy 
ct Ospedale Spirito Santo di Pescara, Geriatria, Italy

${ }^{\mathrm{cu}}$ Ospedale San Giuseppe, Empoli, USL Toscana Centro, Firenze, Medicina Interna I, Italy

${ }^{\mathrm{cv}}$ ASST Fatebenefratelli - Sacco, Milano, Medicina Interna a indirizzo Pneumologico, Italy
${ }^{\mathrm{cw}}$ Azienda Ospedaliera Universitaria di Parma, Clinica e Terapia Medica, Italy

${ }^{\mathrm{cx}}$ Ospedale San Giuseppe Multimedica Spa, U.O. Medicina Generale, Italy E-mail address: carlotta.franchi@marionegri.it (F. Carlotta).

* Corresponding author at: Unit of Pharmacoepidemiological Research in Older People, Istituto di Ricerche Farmacologiche Mario Negri IRCCS, Via Mario Negri, 2, 20156 Milano, Italy. 